Mathematical Modelling And Analysis

Volume 19 Number 1, February 2014, 127-143

http://dx.doi.org/10.3846/13926292.2014.893457

(c) Vilnius Gediminas Technical University, 2014
Publisher: Taylor\&Francis and VGTU

http://www.tandfonline.com/TMMA

Print ISSN: 1392-6292

Online ISSN: 1648-3510

\title{
Asymptotic Expansions for Approximate Solutions of Hammerstein Integral Equations with Green's Function Type Kernels
}

\author{
Rekha P. Kulkarni and Akshay S. Rane \\ Indian Institute of Technology Bombay \\ Powai, 400076 Mumbai, India \\ E-mail: rpk@math.iitb.ac.in \\ E-mail(corresp.): akshayrane11@gmail.com
}

Received August 27, 2013; revised January 26, 2014; published online February 20, 2014

\begin{abstract}
We consider approximation of a nonlinear Hammerstein equation with a kernel of the type of Green's function using the Nyström method based on the composite midpoint and the composite modified Simpson rules associated with a uniform partition. We obtain asymptotic expansions for the approximate solution $u_{n}$ at the node points as well as at the partition points and use Richardson extrapolation to obtain approximate solutions with higher orders of convergence. Numerical results are presented which confirm the theoretical orders of convergence.
\end{abstract}

Keywords: Hammerstein equation, Green's function type kernels, Nyström method, asymptotic expansion.

AMS Subject Classification: 45B05; 65R20.

\section{Introduction}

Asymptotic error analysis of approximate solutions of operator equations is a classical numerical analysis topic. Well known methods for approximate solutions of operator equations are the Nyström methods and the projection related methods such as the classical Galerkin methods and its variants. These methods are extensively studied in the research literature. On establishing the convergence of the approximate solution to the actual solution, one is often interested in improving the orders of convergence of the approximate solution. If asymptotic expansion for an approximate solution is established, then the Richardson extrapolation can be used to improve the orders of convergence.

Asymptotic expansions for approximate solutions of linear integral equations with smooth kernels, in the case of Nyström methods, has been studied in Baker [3]. The case of asymptotic expansions in the case of the iterated collocation and the iterated Galerkin method has been studied by McLean [7]. 
There are physical problems which give rise to integral equations with nonsmooth kernels, namely the Green's type kernel. Such a kernel lacks differentiability properties along the diagonal. For an integral operator with a Green's kernel, the only case of asymptotic expansion of the approximate solution of a second kind Fredholm integral equation was that considered by Baker [3]. He studied it in the context of a Nyström method with the composite trapezoidal rule. The proof in this case is similar to the case of smooth kernels and uses the classical Euler-MacLaurin summation formula. These results were extended to the case of the Nyström method with the composite midpoint and the composite modified Simpson rule, proposed by Cubillos [4], in Kulkarni and Rane [6]. In the case of the Nyström approximation with the composite midpoint rule, some node points are in the interior of the subintervals of integration involved in the integral operator. As a consequence, the integrand fails to be differentiable at an interior point of the subinterval of integration. Hence an extended version of the Euler-MacLaurin summation formula, for functions which fail to be differentiable at an interior point, is proved in Kulkarni and Rane [6].

The problem of asymptotic expansion for an approximate solution of a nonlinear Hammerstein equation with the kernel of the Green's type, was considered by Ford [5]. He considered it in the case of the Nyström method with the composite trapezoidal rule. The main purpose of this paper is to extend the results of Ford [5] in the case of Nyström method with the composite midpoint rule and the modified Simpson's rule. In developing the asymptotic expansion for nonlinear equations, two things are to be taken in to account namely the nature of the Green's kernel and the nonlinearity of the integral equation. The main points involved in obtaining the asymptotic expansions for the approximate solution at the nodes are the extended Euler-MacLaurin summation formula and the results of Ford [5]. But in the case of the composite midpoint rule, in addition to the node points, we also require the asymptotic expansion of the approximate solution at the partition points, so as to apply the extrapolation technique. Using asymptotic expansion at the node points and the Taylor series expansion, asymptotic expansions at the partition points is obtained.

\section{Preliminaries}

Let $X=C^{0}[0,1]$ denote the space of all continuous functions defined on $[0,1]$ equipped with the supremum norm. We consider the following Hammerstein equation:

$$
u(s)-\int_{0}^{1} k(s, t) \psi(t, u(t)) d t=f(s), \quad 0 \leq s \leq 1
$$

where the kernel $k(\cdot, \cdot) \in C^{0}([0,1] \times[0,1])$. In addition, we assume that

$$
k(s, t)= \begin{cases}k_{1}(s, t) & \text { if } s \leq t \\ k_{2}(s, t) & \text { if } t<s\end{cases}
$$

with $k_{1} \in C^{m+2}(\{0 \leq s \leq t \leq 1\}), k_{2} \in C^{m+2}(\{0 \leq t \leq s \leq 1\})$. Here $m$ is an even positive integer. Thus, for each $s \in[0,1]$, the function $k(s, t)$ is $m+2$ 
times differentiable with respect to $t$ in $[0, s) \cup(s, 1]$. We shall denote the class of such kernels by $\mathcal{C}(m+2,0)$.

We assume that $f \in C^{m+2}[0,1]$ and that $\psi \in C^{m+2}([0,1] \times \mathbb{R})$ satisfies the Lipschitz condition with respect to the second variable, that is, there is a constant $L$ such that

$$
\left|\psi\left(t, t_{1}\right)-\psi\left(t, t_{2}\right)\right| \leq L\left|t_{1}-t_{2}\right|
$$

for all $t_{1}, t_{2} \in \mathbb{R}, t \in[0,1]$. We assume that equation (2.1) has a unique solution. With the assumptions on $k, \psi$ and $f$, the unique solution $u \in C^{m+2}[0,1]$. (See Theorem 2.1 of Sidi [8].) We write (2.1) as

$$
u-T \Psi u=f
$$

where

$$
\begin{aligned}
& (T x)(s)=\int_{0}^{1} k(s, t) x(t) d t, \quad s \in[0,1], x \in C[0,1], \\
& (\Psi x)(t)=\psi(t, x(t)) .
\end{aligned}
$$

The operator $T: C^{0}[0,1] \rightarrow C^{0}[0,1]$ is compact. Let $T_{n}$ be a sequence of continuous finite rank operators converging pointwise to $T$ and let $\left\{T_{n}\right\}$ be collectively compact, that is, the set $\left\{T_{n} x: n \geq 1,\|x\|_{\infty} \leq 1\right\}$ has a compact closure in $X$. The integral equation (2.2) is approximated by

$$
u_{n}-T_{n} \Psi u_{n}=f .
$$

Let $B_{0}(t)=1$ and for $p \geq 1$, let $B_{p}(t)$ denote the Bernoulli polynomial of degree $p$. For $p \geq 0$, we define $\bar{B}_{p}$ as a periodic function on $\mathbb{R}$ with period 1 :

$$
\bar{B}_{p}(t)=B_{p}(t), \quad 0 \leq t<1, \quad \bar{B}_{p}(t+1)=\bar{B}_{p}(t), \quad t \in \mathbb{R} .
$$

We quote below an extension of the Euler-MacLaurin series expansion for functions which are $m$ times differentiable on $[0,1]$ except at one point.

Euler-MacLaurin Series Expansion: (Kulkarni and Rane [6].)

Let $m \geq 2$. Fix $s \in(0,1)$. Let $f:[0,1] \rightarrow \mathbb{R}$ be a continuous function such that

(i) the derivatives of $f, f^{(1)}, f^{(2)}, \ldots, f^{(m)}$ exist on $[0, s) \cup(s, 1]$,

(ii) the $m$-th derivative $f^{(m)}$ is integrable on $[0,1]$,

(iii) for $p=1,2, \ldots, m, f^{(p)}(s+)-f^{(p)}(s-)$ is nonzero and finite.

Then for $0 \leq \tau \leq 1$,

$$
\begin{aligned}
f(\tau)= & \int_{0}^{1} f(t) d t+\sum_{p=1}^{m} \frac{B_{p}(\tau)}{p !}\left[f^{(p-1)}(1-)-f^{(p-1)}(0+)\right] \\
& -\sum_{p=2}^{m} \frac{\bar{B}_{p}(\tau-s)}{p !}\left[f^{(p-1)}(s+)-f^{(p-1)}(s-)\right]+R_{m},
\end{aligned}
$$

where

$$
R_{m}=-\int_{0}^{1} \frac{\bar{B}_{m}(\tau-t)}{m !} f^{(m)}(t) d t
$$




\subsection{Nyström approximation}

Let $n \in \mathbb{N}, h=1 / n$ and

$$
\sum_{j=1}^{N} w_{j} x\left(r_{j}\right) \approx \int_{0}^{1} x(t) d t
$$

be a convergent composite quadrature rule with respect to the uniform partition

$$
0<\frac{1}{n}<\frac{2}{n}<\cdots<\frac{n-1}{n}<1
$$

of $[0,1]$. Here $N$, the weights $w_{j}$ and the nodes $r_{j}$ depend on $n$. The Nyström approximation of $T$ is defined as

$$
\left(T_{n} x\right)(s)=\sum_{j=1}^{N} w_{j} k\left(s, r_{j}\right) x\left(r_{j}\right), \quad s \in[0,1] .
$$

Then $\left\{T_{n}\right\}$ is a collectively compact family of continuous finite rank operators converging to $T$ pointwise. (See Atkinson [1].) Now onwards we assume that $x$ belongs to $C^{m+2}[0,1]$. Let

$$
t_{i}=(i-1) / n=(i-1) h, \quad i=1,2, \ldots, n+1
$$

be the partition points and for $i=1, \ldots, n$

$$
s_{i}=\frac{t_{i}+t_{i+1}}{2}=\left(i-\frac{1}{2}\right) h
$$

be the midpoints of $\left[t_{i}, t_{i+1}\right]$.

In the case of approximation of an integral operator $T$ with the kernel of the type of Green's function by Nyström operator $T_{n}$, asymptotic expansions for $\left(T_{n}-T\right) x$ at the node points are obtained in Kulkarni and Rane [6]. The proofs of these expansions are based on the Euler-MacLaurin series expansion (2.3). As the derivation of the asymptotic expansions for the approximate solutions of the nonlinear equations at the nodes/partition points is based on these expansions, we describe them below.

Composite Midpoint Rule. Let

$$
\left(T_{n} x\right)(s)=h \sum_{j=1}^{n} k\left(s, s_{j}\right) x\left(s_{j}\right), \quad s \in[0,1] .
$$

Then for $i=2,3, \ldots, n$

$$
\left(T_{n} x\right)\left(t_{i}\right)=(T x)\left(t_{i}\right)+\sum_{p=1}^{m / 2}\left(A_{2 p} x\right)\left(t_{i}\right) h^{2 p}+\sum_{p=1}^{m / 2}\left(C_{2 p} x\right)\left(t_{i}\right) h^{2 p}+O\left(h^{m+2}\right),
$$


whereas $i=1$ or $i=n+1$

$$
\left(T_{n} x\right)\left(t_{i}\right)=(T x)\left(t_{i}\right)+\sum_{p=1}^{m / 2}\left(A_{2 p} x\right)\left(t_{i}\right) h^{2 p}+O\left(h^{m+2}\right),
$$

where for $s \in[0,1]$

$$
\begin{aligned}
& \left(A_{2 p} x\right)(s)=\frac{B_{2 p}\left(\frac{1}{2}\right)}{(2 p) !}\left[\left(\frac{\partial}{\partial t}\right)^{2 p-1}(k(s, t) x(t))\right]_{t=0}^{t=1} \\
& \left(C_{2 p} x\right)(s)=-\frac{B_{2 p}\left(\frac{1}{2}\right)}{(2 p) !}\left[\left(\frac{\partial}{\partial t}\right)^{2 p-1}(k(s, t) x(t))\right]_{t=s-}^{t=s+}
\end{aligned}
$$

Also, for $i=1,2, \ldots, n$

$$
\left(T_{n} x\right)\left(s_{i}\right)=(T x)\left(s_{i}\right)+\sum_{p=1}^{m / 2}\left(A_{2 p} x\right)\left(s_{i}\right) h^{2 p}+\sum_{p=1}^{m / 2}\left(\tilde{C}_{2 p} x\right)\left(s_{i}\right) h^{2 p}+O\left(h^{m+2}\right)
$$

where for $s \in[0,1]$

$$
\left(\tilde{C}_{2 p} x\right)(s)=-\frac{B_{2 p}(0)}{(2 p) !}\left[\left(\frac{\partial}{\partial t}\right)^{2 p-1}(k(s, t) x(t))\right]_{t=s-}^{t=s+} .
$$

See, Kulkarni and Rane [6]. Note that, $A_{2 p} x, C_{2 p} x, \tilde{C}_{2 p} x \in C^{m+2-2 p}[0,1]$.

Modified Simpson Rule. From Theorem 5.2 of Kulkarni and Rane [6], we observe that if $T_{n}$ is the Nyström approximation associated with the composite Simpson rule then the asymptotic expansion for $\left(T_{n}-T\right) x$ at the partition points starts from $h^{4}$ onwards whereas at the midpoints it starts from $h^{2}$ onwards. In order to restore the order of convergence of $h^{4}$, we consider the following modified Simpson method. (See Atkinson and Shampine [2], Cubillos [4].) We introduce the following notation:

$$
S\{x, a, b\}=\frac{b-a}{6}\left(x(a)+4 x\left(\frac{a+b}{2}\right)+x(b)\right) .
$$

For $i=1,2, \ldots, n$, let $\left.\tilde{x}\right|_{\left[t_{i}, t_{i+1}\right]}$ be the quadratic polynomial such that

$$
\tilde{x}\left(t_{i}\right)=x\left(t_{i}\right), \quad \tilde{x}\left(s_{i}\right)=x\left(s_{i}\right), \quad \tilde{x}\left(t_{i+1}\right)=x\left(t_{i+1}\right) .
$$

Then $\tilde{x}:[0,1] \rightarrow \mathbb{R}$ is a continuous piecewise quadratic polynomial with respect to the uniform partition (2.4).

For $s \in[0,1]$, we define

$$
\left(T_{n} x\right)(s)=\sum_{i=1}^{n}\left(S\left\{k(s, \cdot) \tilde{x}(\cdot), t_{i}, s_{i}\right\}+S\left\{k(s, \cdot) \tilde{x}(\cdot), s_{i}, t_{i+1}\right\}\right) .
$$


Let

$$
r_{2 j-1}=t_{j}, \quad j=1,2, \ldots, n+1, \quad r_{2 j}=s_{j}, \quad j=1,2, \ldots, n .
$$

Note that the formula for $\left(T_{n} x\right)$ can be written as

$$
\begin{aligned}
& \left(T_{n} x\right)(s)=\sum_{j=1}^{2 n+1} w_{j}(s) x\left(r_{j}\right), \quad s \in[0,1], \\
& w_{1}(s)=\frac{h}{12}\left[k\left(s, t_{1}\right)+\frac{3}{2} k\left(s, \frac{t_{1}+s_{1}}{2}\right)-\frac{1}{2} k\left(s, \frac{s_{1}+t_{2}}{2}\right)\right],
\end{aligned}
$$

for $j=1, \ldots, n$

$$
w_{2 j}(s)=\frac{h}{12}\left[3 k\left(s, \frac{t_{j}+s_{j}}{2}\right)+2 k\left(s, s_{j}\right)+3 k\left(s, \frac{s_{j}+t_{j+1}}{2}\right)\right],
$$

for $j=2,3, \ldots, n$

$$
\begin{aligned}
w_{2 j-1}(s)= & \frac{h}{12}\left[2 k\left(s, t_{j}\right)+\frac{3}{2} k\left(s, \frac{t_{j}+s_{j}}{2}\right)-\frac{1}{2} k\left(s, \frac{s_{j}+t_{j+1}}{2}\right)\right] \\
& +\frac{h}{12}\left[\frac{3}{2} k\left(s, \frac{s_{j-1}+t_{j}}{2}\right)-\frac{1}{2} k\left(s, \frac{t_{j-1}+s_{j-1}}{2}\right)\right], \\
w_{2 n+1}(s)= & \frac{h}{12}\left[-\frac{1}{2} k\left(s, \frac{t_{n}+s_{n}}{2}\right)+\frac{3}{2} k\left(s, \frac{s_{n}+t_{n+1}}{2}\right)+k\left(s, t_{n+1}\right)\right] .
\end{aligned}
$$

Note that $T_{n}$ is a collectively compact family of operators converging to $T$ pointwise (see Cubillos [4].)

The following asymptotic expansion at the node points is proved in Kulkarni and Rane [6]:

$$
\left(T_{n} x\right)\left(r_{i}\right)=(T x)\left(r_{i}\right)+\sum_{p=2}^{m / 2}\left(A_{2 p} x\right)\left(r_{i}\right)\left(\frac{h}{2}\right)^{2 p}+\sum_{p=2}^{m / 2}\left(C_{2 p} x\right)\left(r_{i}\right)\left(\frac{h}{2}\right)^{2 p}+O\left(h^{m+2}\right)
$$

where for $s \in[0,1]$

$$
\begin{aligned}
\left(A_{2 p} x\right)(s)= & \frac{1}{(2 p) !}\left(\frac{B_{2 p}(0)+4 B_{2 p}\left(\frac{1}{2}\right)+B_{2 p}(1)}{6}\right)\left[\left(\frac{\partial}{\partial t}\right)^{2 p-1}(k(s, t) x(t))\right]_{t=0}^{t=1}, \\
\left(C_{2 p} x\right)(s)= & -\frac{1}{(2 p) !}\left(\frac{B_{2 p}(0)+4 B_{2 p}\left(\frac{1}{2}\right)+B_{2 p}(1)}{6}\right) \\
& \times\left[\left(\frac{\partial}{\partial t}\right)^{2 p-1}(k(s, t) x(t))\right]_{t=s-}^{t=s+} .
\end{aligned}
$$

\section{Main Results}

In this section we shall briefly describe two new methods and derive asymptotic expansions for the approximate solution $u_{n}$, in the following two cases. 


\section{a) Nyström Method, Composite Midpoint Rule:}

Using the Nyström approximation associated with the composite midpoint rule (2.5), the nonlinear integral equation (2.1) is approximated by

$$
u_{n}(s)-h \sum_{j=1}^{n} k\left(s, s_{j}\right) \psi\left(s_{j}, u_{n}\left(s_{j}\right)\right)=f(s), \quad s \in[0,1] .
$$

Evaluating the above equation at $s=s_{i}, i=1,2, \ldots, n$, we obtain a system of $n$ nonlinear equations.

\section{b) Nyström Method, Modified Simpson Rule:}

Using the Modified Simpson rule (2.8), the nonlinear integral equation (2.1) is approximated by

$$
u_{n}(s)-\sum_{j=1}^{2 n+1} w_{j}(s) \psi\left(r_{j}, u_{n}\left(r_{j}\right)\right)=f(s), \quad s \in[0,1] .
$$

Evaluating the above equation at $s=r_{i}, i=1,2, \ldots, 2 n+1$, we obtain a system of $2 n+1$ nonlinear equations. As in the proof of Theorem 2.4 of Ford [5], it can be proved that equations (3.1) and (3.2) have a unique solution for $n$ large enough. The proof of the following Theorem on asymptotic expansions of $u_{n}-u$ at the node points in the case of the composite midpoint and the modified Simpson rule is similar to that of Theorem 4.2 of Ford et al [5], we only state the result.

Theorem 1. Let $T$ be an integral operator with the kernel $k(\cdot, \cdot) \in \mathcal{C}(m+2,0)$. Let $u$ be a solution of the operator equation $u-T \Psi u=f$, where $f \in C^{m+2}[0,1]$, $\psi \in C^{m+2}([0,1] \times \mathbb{R})$ satisfies the Lipschitz condition with respect to the second variable. Assume that 1 is not an eigenvalue of $(T \psi)^{\prime}(u)$, where the prime notation denotes the Fréchet derivative.

a) If $T_{n}$ is the Nyström approximation associated with the composite midpoint rule, then for sufficiently large $n, u_{n}$ has an asymptotic expansion

$$
u_{n}\left(s_{i}\right)=u\left(s_{i}\right)+\sum_{p=1}^{m / 2} \eta_{2 p}\left(s_{i}\right) h^{2 p}+O\left(h^{m+2}\right), \quad i=1, \ldots, n,
$$

where $\eta_{2 p} \in C^{m+2-2 p}[0,1], p=1, \ldots, m / 2$ are independent of $h$.

b) If $T_{n}$ is the Nyström approximation associated with the composite modified Simpson rule then for sufficiently large $n, u_{n}$ has an asymptotic expansion

$$
u_{n}\left(s_{i}\right)=u\left(s_{i}\right)+\sum_{p=2}^{m / 2} \eta_{2 p}\left(s_{i}\right)\left(\frac{h}{2}\right)^{2 p}+O\left(h^{m+2}\right), \quad i=1, \ldots, n
$$

and

$$
u_{n}\left(t_{i}\right)=u\left(t_{i}\right)+\sum_{p=2}^{m / 2} \eta_{2 p}\left(t_{i}\right)\left(\frac{h}{2}\right)^{2 p}+O\left(h^{m+2}\right), \quad i=1, \ldots, n+1,
$$

where functions $\eta_{2 p} \in C^{m+2-2 p}[0,1], p=2, \ldots, m / 2$ are independent of $h$. 
The purpose of asymptotic expansions is to obtain higher order approximations using the Richardson extrapolation. Consider the case of the composite midpoint rule. Let $S^{(n)}=\left\{s_{i}^{(n)}: i=1, \ldots, n\right\}$ denote the set of node points with respect to the partition with $n$ intervals and $S^{(2 n)}=\left\{s_{i}^{(2 n)}: i=1, \ldots, 2 n\right\}$ denote the set of node points obtained by subdividing each subintervals in two equal parts. Then $S^{(n)} \cap S^{(2 n)}=\emptyset$, and hence we cannot use the extrapolation technique. However, we obtain below asymptotic expansions for $u_{n}$ at the partition points $t_{i}$ so that Richardson extrapolation is applicable.

For $l=1, \ldots, m+1$, let $\psi^{(0, l)}(t, \cdot)$ denote the $l$-th partial derivative of $\psi(t, \cdot)$ with respect to the second variable. We now prove a Proposition before proving the main result.

Proposition 1. Let $T_{n}$ be the Nyström approximation associated with the composite midpoint rule. For $1 \leq l \leq m+1$, define

$$
\left(\Psi^{[l]} u\right)(t)=\psi^{(0, l)}(t, u(t)), \quad t \in[0,1] .
$$

For $1 \leq p \leq m / 2$, let

$$
\xi_{2 p, l}=\sum_{\alpha_{1}+\cdots+\alpha_{l}=p} \eta_{2 \alpha_{1}} \ldots \eta_{2 \alpha_{l}}, \quad l \leq p \leq m / 2 .
$$

Then for $s \in[0,1]$

$$
\left(T_{n} \Psi u_{n}\right)(s)=\left(T_{n} \Psi u\right)(s)+\sum_{p=1}^{m / 2}\left(\sum_{l=1}^{p} \frac{T_{n}\left(\left(\Psi^{[l]} u\right) \xi_{2 p, l}\right)(s)}{l !}\right) h^{2 p}+O\left(h^{m+2}\right) .
$$

Proof. By Taylor's Theorem,

$$
\begin{aligned}
\psi\left(t, u_{n}(t)\right)= & \psi(t, u(t))+\sum_{l=1}^{m+1} \frac{\psi^{(0, l)}(t, u(t))\left(u_{n}(t)-u(t)\right)^{l}}{l !} \\
& +O\left(\left(u_{n}(t)-u(t)\right)^{m+2}\right), \quad t \in[0,1] .
\end{aligned}
$$

As in Theorem 2.4 of Ford [5], it can be proved that $\left\|u_{n}-u\right\|_{\infty}=O\left(h^{2}\right)$. Then

$$
\left\|u_{n}-u\right\|_{\infty}^{m+2}=O\left(h^{2 m+4}\right) .
$$

Hence for $j=1, \ldots, n$,

$$
\begin{aligned}
\psi\left(s_{j}, u_{n}\left(s_{j}\right)\right)= & \psi\left(s_{j}, u\left(s_{j}\right)\right)+\sum_{l=1}^{m+1} \frac{\psi^{(0, l)}\left(s_{j}, u\left(s_{j}\right)\right)\left(u_{n}\left(s_{j}\right)-u\left(s_{j}\right)\right)^{l}}{l !} \\
& +O\left(h^{2 m+4}\right) .
\end{aligned}
$$

From (3.3), we have

$$
u_{n}\left(s_{j}\right)-u\left(s_{j}\right)=\sum_{r=1}^{m / 2} \eta_{2 r}\left(s_{j}\right) h^{2 r}+O\left(h^{m+2}\right)
$$


Hence for $1 \leq l \leq m+1$,

$$
\begin{aligned}
\left(u_{n}\left(s_{j}\right)-u\left(s_{j}\right)\right)^{l} & =\left(\sum_{r=1}^{m / 2} \eta_{2 r}\left(s_{j}\right) h^{2 r}+O\left(h^{m+2}\right)\right)^{l} \\
& =\sum_{p=l}^{m / 2} \xi_{2 p, l}\left(s_{j}\right) h^{2 p}+O\left(h^{m+2}\right)
\end{aligned}
$$

where $\xi_{2 p, l}$ is given by (3.4). Substituting the above expression in (3.6) and rearranging terms, we get

$$
\psi\left(s_{j}, u_{n}\left(s_{j}\right)\right)=\psi\left(s_{j}, u\left(s_{j}\right)\right)+\sum_{p=1}^{m / 2}\left(\sum_{l=1}^{p} \frac{\psi^{(0, l)}\left(s_{j}, u\left(s_{j}\right)\right) \xi_{2 p, l}\left(s_{j}\right)}{l !}\right) h^{2 p}+O\left(h^{m+2}\right) .
$$

Multiplying the above equation by $h k\left(s, s_{j}\right)$ and taking the sum from $j=$ $1, \ldots, n$, we obtain

$$
\begin{aligned}
& h \sum_{j=1}^{n} k\left(s, s_{j}\right) \psi\left(s_{j}, u_{n}\left(s_{j}\right)\right)=h \sum_{j=1}^{n} k\left(s, s_{j}\right) \psi\left(s_{j}, u\left(s_{j}\right)\right)+\sum_{p=1}^{m / 2} \\
& \quad \times\left(\sum_{l=1}^{p} \frac{1}{l !} h \sum_{j=1}^{n} k\left(s, s_{j}\right) \psi^{(0, l)}\left(s_{j}, u\left(s_{j}\right)\right) \xi_{2 p, l}\left(s_{j}\right)\right) h^{2 p}+O\left(h^{m+2}\right), \quad s \in[0,1] .
\end{aligned}
$$

Thus, for $s \in[0,1]$,

$$
\left(T_{n} \Psi u_{n}\right)(s)=\left(T_{n} \Psi u\right)(s)+\sum_{p=1}^{m / 2}\left(\sum_{l=1}^{p} \frac{T_{n}\left(\left(\Psi^{[l]} u\right) \xi_{2 p, l}\right)(s)}{l !}\right) h^{2 p}+O\left(h^{m+2}\right),
$$

which completes the proof.

Now we prove the main result concerning asymptotic expansions for $u_{n}$ at the partition points.

Theorem 2. Let $T_{n}$ be the Nyström approximation associated with the composite midpoint rule. For $i=2, \ldots, n$, we have

$$
u_{n}\left(t_{i}\right)=u\left(t_{i}\right)+\sum_{p=1}^{m / 2} \delta_{2 p}\left(t_{i}\right) h^{2 p}+O\left(h^{m+2}\right),
$$

where functions $\delta_{2 p} \in C^{m+2-2 p}[0,1]$ are independent of $h$.

For $i=1$ and $i=n+1$, we have

$$
u_{n}\left(t_{i}\right)=u\left(t_{i}\right)+\sum_{p=1}^{m / 2} \tilde{\delta}_{2 p}\left(t_{i}\right) h^{2 p}+O\left(h^{m+2}\right),
$$

where functions $\tilde{\delta}_{2 p} \in C^{m+2-2 p}[0,1]$ are independent of $h$. 
Proof. Since

$$
u_{n}-T_{n} \Psi u_{n}=f
$$

we have

$$
u_{n}\left(t_{i}\right)=\left(T_{n} \Psi u_{n}\right)\left(t_{i}\right)+f\left(t_{i}\right), \quad i=1, \ldots, n+1 .
$$

Substituting for $\left(T_{n} \Psi u_{n}\right)\left(t_{i}\right)$, from (3.5) in the above equation, we get

$$
\begin{aligned}
u_{n}\left(t_{i}\right)= & \left(T_{n} \Psi u\right)\left(t_{i}\right)+f\left(t_{i}\right)+\sum_{p=1}^{m / 2}\left(\sum_{l=1}^{p} \frac{T_{n}\left(\left(\Psi^{[l]} u\right) \xi_{2 p, l}\right)\left(t_{i}\right)}{l !}\right) h^{2 p} \\
& +O\left(h^{m+2}\right), \quad i=1, \ldots, n+1 .
\end{aligned}
$$

Note that for $1 \leq p \leq m / 2$ and $1 \leq l \leq p$,

$$
\left(\Psi^{[l]} u\right) \xi_{2 p, l} \in C^{m+2-2 p}[0,1] .
$$

For $x \in C^{m+2}[0,1]$ from $(2.6),(2.7)$ we have

$$
\left(T_{n} x\right)\left(t_{i}\right)=(T x)\left(t_{i}\right)+\sum_{p=1}^{m / 2} \beta_{2 p}(x)\left(t_{i}\right) h^{2 p}+O\left(h^{m+2}\right), \quad i=2, \ldots, n,
$$

whereas $i=1$ or $i=n+1$

$$
\left(T_{n} x\right)\left(t_{i}\right)=(T x)\left(t_{i}\right)+\sum_{p=1}^{m / 2} \gamma_{2 p}(x)\left(t_{i}\right) h^{2 p}+O\left(h^{m+2}\right),
$$

where for $p=1,2, \ldots, m / 2$

$$
\beta_{2 p}(x)=A_{2 p} x+C_{2 p} x \quad \text { and } \quad \gamma_{2 p}(x)=A_{2 p} x .
$$

Then $\beta_{2 p}(x), \gamma_{2 p}(x) \in C^{m+2-2 p}[0,1]$. Thus

$$
\begin{aligned}
\left(T_{n} \Psi u\right)\left(t_{i}\right)= & (T \Psi u)\left(t_{i}\right)+\sum_{p=1}^{m / 2} \beta_{2 p}(\Psi u)\left(t_{i}\right) h^{2 p} \\
& +O\left(h^{m+2}\right), \quad i=2, \ldots, n
\end{aligned}
$$

and for $1 \leq p \leq(m / 2), 1 \leq l \leq p$

$$
\begin{aligned}
T_{n}\left(\left(\Psi^{[l]} u\right) \xi_{2 p, l}\right)\left(t_{i}\right)= & T\left(\left(\Psi^{[l]} u\right) \xi_{2 p, l}\right)\left(t_{i}\right)+\sum_{q=1}^{(m / 2)-p} \beta_{2 q}\left(\left(\Psi^{[l]} u\right) \xi_{2 p, l}\right)\left(t_{i}\right) h^{2 q} \\
& +O\left(h^{m+2-2 p}\right), \quad i=2, \ldots, n .
\end{aligned}
$$

Substituting (3.10) and (3.11) in (3.9), for $i=2, \ldots, n$, we obtain

$$
\begin{aligned}
u_{n}\left(t_{i}\right)= & (T \Psi u)\left(t_{i}\right)+f\left(t_{i}\right) \\
& +\sum_{p=1}^{m / 2} \beta_{2 p}(\Psi u)\left(t_{i}\right) h^{2 p}+\sum_{p=1}^{m / 2} \sum_{l=1}^{p} \frac{1}{l !} T\left(\left(\Psi^{[l]} u\right) \xi_{2 p, l}\right)\left(t_{i}\right) h^{2 p} \\
& +\sum_{p=1}^{(m / 2)-1} \sum_{l=1}^{p} \sum_{q=1}^{(m / 2)-p} \frac{1}{l !} \beta_{2 q}\left(\left(\Psi^{[l]} u\right) \xi_{2 p, l}\right)\left(t_{i}\right) h^{2 p+2 q}+O\left(h^{m+2}\right) .
\end{aligned}
$$


Using $u=T \Psi u+f$ and rearranging terms, we obtain

$$
\begin{aligned}
u_{n}\left(t_{i}\right)= & u\left(t_{i}\right)+\sum_{p=1}^{m / 2} \beta_{2 p}(\Psi u)\left(t_{i}\right) h^{2 p}+\sum_{p=1}^{m / 2} \sum_{l=1}^{p} \frac{1}{l !} T\left(\left(\Psi^{[l]} u\right) \xi_{2 p, l}\right)\left(t_{i}\right) h^{2 p} \\
& +\sum_{p=2}^{m / 2}\left(\sum_{l=1}^{p-1} \sum_{q=1}^{p-l} \frac{1}{l !} \beta_{2 q}\left(\left(\Psi^{[l]} u\right) \xi_{2 p-2 q, l}\right)\left(t_{i}\right)\right) h^{2 p}+O\left(h^{m+2}\right) .
\end{aligned}
$$

Thus for $i=2, \ldots, n$

$$
\begin{aligned}
u_{n}\left(t_{i}\right)= & u\left(t_{i}\right)+\left(\beta_{2}(\Psi u)\left(t_{i}\right)+T\left(\left(\Psi^{[1]} u\right) \xi_{2,1}\right)\left(t_{i}\right)\right) h^{2} \\
& +\sum_{p=2}^{m / 2}\left(\beta_{2 p}(\Psi u)\left(t_{i}\right)+\sum_{l=1}^{p} \frac{1}{l !} T\left(\left(\Psi^{[l]} u\right) \xi_{2 p, l}\right)\left(t_{i}\right)\right) h^{2 p} \\
& +\sum_{p=2}^{m / 2}\left(\sum_{l=1}^{p-1} \sum_{q=1}^{p-l} \frac{1}{l !} \beta_{2 q}\left(\left(\Psi^{[l]} u\right) \xi_{2 p-2 q, l}\right)\left(t_{i}\right)\right) h^{2 p}+O\left(h^{m+2}\right) \\
= & u\left(t_{i}\right)+\sum_{p=1}^{m / 2} \delta_{2 p}\left(t_{i}\right) h^{2 p}+O\left(h^{m+2}\right)
\end{aligned}
$$

where $\delta_{2}=\beta_{2}(\Psi u)+T\left(\left(\Psi^{[1]} u\right) \xi_{2,1}\right)$ and for $p=2,3, \ldots, m / 2$

$$
\delta_{2 p}=\beta_{2 p}(\Psi u)+\sum_{l=1}^{p} \frac{1}{l !} T\left(\left(\Psi^{[l]} u\right) \xi_{2 p, l}\right)+\sum_{l=1}^{p-1} \sum_{q=1}^{p-l} \frac{1}{l !} \beta_{2 q}\left(\left(\Psi^{[l]} u\right) \xi_{2 p-2 q, l}\right) .
$$

This proves (3.7). The proof of (3.8) is similar.

\section{Extrapolation}

In this section, we apply Richardson extrapolation to obtain better orders of convergence for $u_{n}$ at the partition points in the case of the composite midpoint rule and at the node points in the case of composite modified Simpson rule. We first tabulate the results obtained in Theorems 1 and 2 for the asymptotic expansion of $u_{n}$.

1. Nyström Method: Composite Midpoint Rule

$$
\begin{aligned}
& u_{n}\left(t_{i}\right)=u\left(t_{i}\right)+\sum_{p=1}^{m / 2} \delta_{2 p}\left(t_{i}\right) h^{2 p}+O\left(h^{m+2}\right), \\
& u_{n}\left(s_{i}\right)=u\left(s_{i}\right)+\sum_{p=1}^{m / 2} \eta_{2 p}\left(s_{i}\right) h^{2 p}+O\left(h^{m+2}\right) .
\end{aligned}
$$


2. Nyström Method: Composite Modified Simpson Rule

$$
\begin{aligned}
& u_{n}\left(t_{i}\right)=u\left(t_{i}\right)+\sum_{p=2}^{m / 2} \eta_{2 p}\left(t_{i}\right)\left(\frac{h}{2}\right)^{2 p}+O\left(h^{m+2}\right), \\
& u_{n}\left(s_{i}\right)=u\left(s_{i}\right)+\sum_{p=2}^{m / 2} \eta_{2 p}\left(s_{i}\right)\left(\frac{h}{2}\right)^{2 p}+O\left(h^{m+2}\right) .
\end{aligned}
$$

Note that the functions $\eta_{2 p} \in C^{m+2-2 p}[0,1]$ and $\delta_{2 p} \in C^{m+2-2 p}[0,1]$ and are independent of $h$.

For each $t_{i}=\frac{i-1}{n}, i=1, \ldots, n+1$, and for $u_{n}\left(t_{i}\right)$ satisfying (4.1), define $u_{n, 0}\left(t_{i}\right)=u_{n}\left(t_{i}\right)$ and

$$
u_{n, l}\left(t_{i}\right)=\frac{2^{2 l} u_{2 n, l-1}\left(t_{i}\right)-u_{n, l-1}\left(t_{i}\right)}{2^{2 l}-1}, \quad l=1,2, \ldots, m / 2-1 .
$$

Then we have the following result.

Corollary 1. Suppose that the conditions of Theorem 1 hold. Then

$$
u_{n, l}\left(t_{i}\right)=u\left(t_{i}\right)+\sum_{p=l+1}^{m / 2} e_{l, p}\left(t_{i}\right) h^{2 p}+O\left(h^{m+2}\right), \quad i=1,2, \ldots, n+1,
$$

where the functions $e_{l, p}$ are independent of $h$.

In a similar fashion, for each $t_{i}=\frac{i-1}{n}, i=1, \ldots, n+1$, and for $u_{n}\left(t_{i}\right)$ satisfying (4.3) define $u_{n, 0}\left(t_{i}\right)=u_{n}\left(t_{i}\right)$ and

$$
u_{n, l}\left(t_{i}\right)=\frac{2^{2 l+2} u_{2 n, l-1}\left(t_{i}\right)-u_{n, l-1}\left(t_{i}\right)}{2^{2 l+2}-1}, \quad l=1,2, \ldots, m / 2-1 .
$$

For each $s_{i}=\left(i-\frac{1}{2}\right) h, i=1, \ldots, n$, and for $u_{n}\left(s_{i}\right)$ satisfying (4.4) define $u_{n, 0}\left(s_{i}\right)=u_{n}\left(s_{i}\right)$ and

$$
u_{n, l}\left(s_{i}\right)=\frac{2^{2 l+2} u_{2 n, l-1}\left(s_{i}\right)-u_{n, l-1}\left(s_{i}\right)}{2^{2 l+2}-1}, \quad l=1,2, \ldots, m / 2-1 .
$$

Then we have the following result.

Corollary 2. Suppose that the conditions of Theorem 1 hold. Then

$$
\begin{aligned}
& u_{n, l}\left(t_{i}\right)=u\left(t_{i}\right)+\sum_{p=l+2}^{m / 2} e_{l, p}\left(t_{i}\right)\left(\frac{h}{2}\right)^{2 p}+O\left(h^{m+2}\right), \quad i=1,2, \ldots, n+1, \\
& u_{n, l}\left(s_{i}\right)=u\left(s_{i}\right)+\sum_{p=l+2}^{m / 2} e_{l, p}\left(s_{i}\right)\left(\frac{h}{2}\right)^{2 p}+O\left(h^{m+2}\right), \quad i=1,2, \ldots, n,
\end{aligned}
$$

where the functions $e_{l, p}$ are independent of $h$. 


\section{$5 \quad$ Numerical Results}

Consider the following equation

$$
u(s)-\int_{0}^{1} k(s, t) \psi(t, u(t)) d t=f(s), \quad 0 \leq s \leq 1,
$$

where

$$
k(s, t)=\frac{1}{\gamma \sinh \gamma} \begin{cases}\sinh \gamma s \sinh \gamma(1-t) & \text { if } s \leq t \\ \sinh \gamma(1-s) \sinh \gamma t & \text { if } t<s\end{cases}
$$

with $\gamma=\sqrt{12}$,

$$
(\Psi u)(t)=\psi(t, u(t))=\gamma^{2} u(t)-2(u(t))^{3}, \quad t \in[0,1]
$$

and

$$
f(s)=\frac{1}{\sinh \gamma}\left\{2 \sinh \gamma(1-s)+\frac{2}{3} \sinh \gamma s\right\} .
$$

The exact solution is $u(s)=1 /(s+1 / 2)$.

\subsection{Composite midpoint rule}

Equation (5.1) is approximated by

$$
u_{n}(s)-h \sum_{j=1}^{n} k\left(s, s_{j}\right)\left[\gamma^{2} u_{n}\left(s_{j}\right)-2\left(u_{n}\left(s_{j}\right)\right)^{3}\right]=f(s), \quad 0 \leq s \leq 1 .
$$

The solution of the above equation is obtained by solving the nonlinear system of equations

$$
u_{n}\left(s_{i}\right)-h \sum_{j=1}^{n} k\left(s_{i}, s_{j}\right)\left[\gamma^{2} u_{n}\left(s_{j}\right)-2\left(u_{n}\left(s_{j}\right)\right)^{3}\right]=f\left(s_{i}\right), \quad i=1,2, \ldots, n
$$

and then for $s \in[0,1]$

$$
u_{n}(s)=f(s)+h \sum_{j=1}^{n} k\left(s, s_{j}\right)\left[\gamma^{2} u_{n}\left(s_{j}\right)-2\left(u_{n}\left(s_{j}\right)\right)^{3}\right] .
$$

The Picard iteration is applied to obtain the solution of (5.2):

$$
\begin{aligned}
u_{n}^{0}\left(s_{i}\right) & =f\left(s_{i}\right), \quad i=1,2, \ldots, n, \\
u_{n}^{p+1}\left(s_{i}\right) & =f\left(s_{i}\right)+\left(T_{n} \Psi u_{n}^{p}\right)\left(s_{i}\right), \quad i=1,2, \ldots, n, p=0,1, \ldots
\end{aligned}
$$

We choose $n=20, n=40$ and $n=80$. In the above method, 21 Picard iterations were needed for the difference in the successive iterates to be less than $10^{-12}$.

Let $t_{i}=(i-1) / 20, i=1,2, \ldots, 21$ be the partition points in a uniform partition with step size $h=1 / 20$. 
We have

$$
E_{1}^{n}\left(t_{i}\right)=\left|u\left(t_{i}\right)-u_{n}\left(t_{i}\right)\right|=O\left(h^{2}\right), \quad i=1,2, \ldots, 21 .
$$

Define

$$
u_{n, 1}\left(t_{i}\right)=\frac{4 u_{2 n}\left(t_{i}\right)-u_{n}\left(t_{i}\right)}{3}, \quad i=1,2, \ldots, 21 .
$$

Then

$$
E_{2}^{n}\left(t_{i}\right)=\left|u\left(t_{i}\right)-u_{n, 1}\left(t_{i}\right)\right|=O\left(h^{4}\right), \quad i=1,2, \ldots, 21 .
$$

We compute the orders of convergence by the following formula:

$$
\begin{aligned}
& \alpha_{1}=\frac{\log \left(E_{1}^{n}\left(t_{i}\right) / E_{1}^{2 n}\left(t_{i}\right)\right)}{\log (2)}, \quad \beta=\frac{\log \left(E_{2}^{n}\left(t_{i}\right) / E_{2}^{2 n}\left(t_{i}\right)\right)}{\log (2)}, \quad \text { with } n=20, \\
& \alpha_{2}=\frac{\log \left(E_{1}^{n}\left(t_{i}\right) / E_{1}^{2 n}\left(t_{i}\right)\right)}{\log (2)}, \quad \text { with } n=40 .
\end{aligned}
$$

We expect $\alpha_{1}=\alpha_{2}=2$ and $\beta=4$. (See (4.1) and Corollary 1.)

In Tables 1, 2 we give some numerical results which validate the above orders of convergence.

Table 1. Errors and convergence orders for the composite midpoint rule.

\begin{tabular}{cccccc}
\hline$t_{i}$ & $E_{1}^{n}\left(t_{i}\right): n=20$ & $E_{1}^{n}\left(t_{i}\right): n=40$ & $E_{1}^{n}\left(t_{i}\right): n=80$ & $\alpha_{1}$ & $\alpha_{2}$ \\
\hline 0.1 & $4.52 \times 10^{-4}$ & $1.14 \times 10^{-4}$ & $2.84 \times 10^{-5}$ & 1.99 & 2.00 \\
0.2 & $4.61 \times 10^{-4}$ & $1.16 \times 10^{-4}$ & $2.89 \times 10^{-5}$ & 2.00 & 2.00 \\
0.3 & $2.91 \times 10^{-4}$ & $7.29 \times 10^{-5}$ & $1.82 \times 10^{-5}$ & 2.00 & 2.00 \\
0.4 & $6.90 \times 10^{-5}$ & $1.70 \times 10^{-5}$ & $4.24 \times 10^{-6}$ & 2.02 & 2.00 \\
0.5 & $1.40 \times 10^{-4}$ & $3.53 \times 10^{-5}$ & $8.85 \times 10^{-6}$ & 1.98 & 2.00 \\
0.6 & $2.96 \times 10^{-4}$ & $7.45 \times 10^{-5}$ & $1.86 \times 10^{-5}$ & 1.99 & 2.00 \\
0.7 & $3.76 \times 10^{-4}$ & $9.43 \times 10^{-5}$ & $2.36 \times 10^{-5}$ & 1.99 & 2.00 \\
0.8 & $3.61 \times 10^{-4}$ & $9.06 \times 10^{-5}$ & $2.27 \times 10^{-5}$ & 2.00 & 2.00 \\
0.9 & $2.40 \times 10^{-4}$ & $6.00 \times 10^{-5}$ & $1.50 \times 10^{-5}$ & 2.00 & 2.00 \\
\hline
\end{tabular}

Table 2. Errors and convergence orders for the composite midpoint rule: extrapolation

\begin{tabular}{lccc}
\hline$t_{i}$ & $E_{2}^{n}\left(t_{i}\right): n=20$ & $E_{2}^{n}\left(t_{i}\right): n=40$ & $\beta$ \\
\hline 0.1 & $6.19 \times 10^{-7}$ & $4.06 \times 10^{-8}$ & 3.93 \\
0.2 & $3.31 \times 10^{-7}$ & $2.25 \times 10^{-8}$ & 3.88 \\
0.3 & $4.86 \times 10^{-8}$ & $1.54 \times 10^{-9}$ & 4.98 \\
0.4 & $3.36 \times 10^{-7}$ & $1.98 \times 10^{-8}$ & 4.09 \\
0.5 & $4.98 \times 10^{-7}$ & $3.01 \times 10^{-8}$ & 4.05 \\
0.6 & $5.45 \times 10^{-7}$ & $3.33 \times 10^{-8}$ & 4.03 \\
0.7 & $4.96 \times 10^{-7}$ & $3.04 \times 10^{-8}$ & 4.03 \\
0.8 & $3.75 \times 10^{-7}$ & $2.30 \times 10^{-8}$ & 4.03 \\
0.9 & $2.04 \times 10^{-7}$ & $1.25 \times 10^{-8}$ & 4.02 \\
\hline
\end{tabular}




\subsection{Modified Simpson rule}

Equation (5.1) is approximated by

$$
u_{n}(s)-\sum_{j=1}^{2 n+1} w_{j}(s)\left[\gamma^{2} u_{n}\left(r_{j}\right)-2\left(u_{n}\left(r_{j}\right)\right)^{3}\right]=f(s), \quad 0 \leq s \leq 1,
$$

where $w_{j}(s)$ is defined in (2.8). The solution of the above equation is obtained by solving the nonlinear system of equations

$$
u_{n}\left(r_{i}\right)-\sum_{j=1}^{2 n+1} w_{j}\left(r_{i}\right)\left[\gamma^{2} u_{n}\left(r_{j}\right)-2\left(u_{n}\left(r_{j}\right)\right)^{3}\right]=f\left(r_{i}\right), \quad i=1,2, \ldots 2 n+1
$$

and then for $s \in[0,1]$

$$
u_{n}(s)=f(s)+\sum_{j=1}^{2 n+1} w_{j}(s)\left[\gamma^{2} u_{n}\left(r_{j}\right)-2\left(u_{n}\left(r_{j}\right)\right)^{3}\right] .
$$

The Picard iteration is applied to obtain the solution of (5.3):

$$
\begin{aligned}
& u_{n}^{0}\left(r_{i}\right)=f\left(r_{i}\right), \quad i=1,2, \ldots, 2 n+1 \\
& u_{n}^{p+1}\left(r_{i}\right)=f\left(r_{i}\right)+\left(T_{n} \Psi u_{n}^{p}\right)\left(r_{i}\right), \quad i=1,2, \ldots, 2 n+1, p=0,1, \ldots
\end{aligned}
$$

As in the case of the composite midpoint rule, choose $n=20, n=40$ and $n=80$. In the above method, 21 Picard iterations were needed for the difference in the successive iterates to be less than $10^{-12}$.

Table 3. Errors and convergence orders for the composite modified Simpson Rule rule.

\begin{tabular}{cccccc}
\hline$t_{i}$ & $E_{1}^{n}\left(t_{i}\right): n=20$ & $E_{1}^{n}\left(t_{i}\right): n=40$ & $E_{1}^{n}\left(t_{i}\right): n=80$ & $\alpha_{1}$ & $\alpha_{2}$ \\
\hline 0.1 & $2.21 \times 10^{-5}$ & $1.46 \times 10^{-6}$ & $9.30 \times 10^{-8}$ & 3.92 & 3.98 \\
0.2 & $2.45 \times 10^{-5}$ & $1.62 \times 10^{-6}$ & $1.03 \times 10^{-7}$ & 3.92 & 3.98 \\
0.3 & $2.24 \times 10^{-5}$ & $1.47 \times 10^{-6}$ & $9.34 \times 10^{-8}$ & 3.93 & 3.98 \\
0.4 & $1.94 \times 10^{-5}$ & $1.27 \times 10^{-6}$ & $8.05 \times 10^{-8}$ & 3.93 & 3.98 \\
0.5 & $1.63 \times 10^{-5}$ & $1.07 \times 10^{-6}$ & $6.75 \times 10^{-8}$ & 3.93 & 3.98 \\
0.6 & $1.32 \times 10^{-5}$ & $8.65 \times 10^{-7}$ & $5.48 \times 10^{-8}$ & 3.93 & 3.98 \\
0.7 & $1.02 \times 10^{-5}$ & $6.65 \times 10^{-7}$ & $4.22 \times 10^{-8}$ & 3.93 & 3.98 \\
0.8 & $7.02 \times 10^{-6}$ & $4.59 \times 10^{-7}$ & $2.91 \times 10^{-8}$ & 3.94 & 3.98 \\
0.9 & $3.66 \times 10^{-6}$ & $2.39 \times 10^{-7}$ & $1.52 \times 10^{-8}$ & 3.94 & 3.98 \\
\hline
\end{tabular}

Let $t_{i}=(i-1) / 20, i=1,2, \ldots, 21$ be the partition points in a uniform partition with step size $h=1 / 20$. We have

$$
E_{1}^{n}\left(t_{i}\right)=\left|u\left(t_{i}\right)-u_{n}\left(t_{i}\right)\right|=O\left(h^{4}\right), \quad i=1,2, \ldots, 21 .
$$

Define

$$
u_{n, 1}\left(t_{i}\right)=\frac{16 u_{2 n}\left(t_{i}\right)-u_{n}\left(t_{i}\right)}{15}, \quad i=1,2, \ldots, 21
$$


Then

$$
E_{2}^{n}\left(t_{i}\right)=\left|u\left(t_{i}\right)-u_{n, 1}\left(t_{i}\right)\right|=O\left(h^{6}\right), \quad i=1,2, \ldots, 21 .
$$

The orders of convergence $\alpha_{1}, \alpha_{2}$ and $\beta$ are computed as in the case of the composite midpoint rule and presented in Table 3,4 . In the present case, we expect $\alpha_{1}=\alpha_{2}=4$ and $\beta=6$. (See (4.3) and Corollary 2.)

Table 4. Errors and convergence orders for the modified Simpson rule: extrapolation

\begin{tabular}{cccc}
\hline$t_{i}$ & $E_{2}^{n}\left(t_{i}\right): n=20$ & $E_{2}^{n}\left(t_{i}\right): n=40$ & $\beta$ \\
\hline 0.1 & $8.91 \times 10^{-8}$ & $1.57 \times 10^{-9}$ & 5.83 \\
0.2 & $9.10 \times 10^{-8}$ & $1.60 \times 10^{-9}$ & 5.83 \\
0.3 & $7.80 \times 10^{-8}$ & $1.38 \times 10^{-9}$ & 5.82 \\
0.4 & $6.44 \times 10^{-8}$ & $1.16 \times 10^{-9}$ & 5.79 \\
0.5 & $5.24 \times 10^{-8}$ & $9.74 \times 10^{-10}$ & 5.75 \\
0.6 & $4.17 \times 10^{-8}$ & $8.00 \times 10^{-10}$ & 5.70 \\
0.7 & $3.16 \times 10^{-8}$ & $6.26 \times 10^{-10}$ & 5.66 \\
0.8 & $2.15 \times 10^{-8}$ & $4.39 \times 10^{-10}$ & 5.62 \\
0.9 & $1.11 \times 10^{-8}$ & $2.31 \times 10^{-10}$ & 5.58 \\
\hline
\end{tabular}

\section{Conclusions}

Thus it was possible to obtain asymptotic expansions for approximate solutions of a nonlinear Hammerstein equations with Green's kernel, in the context of Nyström methods with the composite midpoint and the composite modified Simpson's rule. We feel that these results can be extended to the iterated collocation method with piecewise constant and piecewise linear polynomials.

\section{Acknowledgments}

The work of R.P. Kulkarni was partially supported by CEFIPRA, Project No. 4101-1. The work of A.S. Rane was supported by a C.S.I.R., India fellowship.

\section{References}

[1] K. Atkinson. The Numerical Solutions of Integral Equations of the Second Kind. Cambridge University Press, Cambridge, UK, 1997.

[2] K. Atkinson and L. Shampine. Solving fredholm integral equations of the second kind in matlab. ACM Trans. Math. Software, 34(4):1-20, 2008. http://dx.doi.org/10.1145/1377596.1377601.

[3] C. Baker. The Numerical Treatment of Integral Equations. Oxford University Press, Oxford, 1977.

[4] P. Cubillos. Integral operators with Green's function type kernels. J. Comput. Appl. Math., 10(1):25-31, 1984.

[5] W. Ford, J. Penniline, Y. Xu and Y. Zhao. Asymptotic error analysis of a quadrature method for integral equations with Green's function kernels. J. Integral Equations Appl., 12(4):349-384, 2000. http://dx.doi.org/10.1216/jiea/1020282235. 
[6] R. Kulkarni and A. Rane. Asymptotic expansions for approximate solutions of Fredholm integral equations with Green's function type kernels. J. Integral Equations Appl., 24(1):39-79, 2012. http://dx.doi.org/10.1216/JIE-2012-24-1-39.

[7] W. Mclean. Asymptotic error expansions for numerical solution of integral equations. IMA J. Numer. Anal., 9(3):373-384, 1989.

[8] A. Sidi and J. Pennline. Improving the accuracy of quadrature method solutions of Fredholm integral equations that arise from nonlinear two-point boundary value problems. J. Integral Equations Appl., 11(1):103-139, 1999.

http://dx.doi.org/10.1216/jiea/1181074263. 